\title{
Process-based functions for seed retention on animals: a test of improved descriptions of dispersal using multiple data sets
}

\author{
James M. Bullock, Stephen J. Galsworthy, Pablo Manzano, Peter Poschlod, Carsten Eichberg, \\ Katherine Walker and Matthias C. Wichmann
}

J. M. Bullock (jmbul@ceh.ac.uk), K. Walker, M. C. Wichmann, Centre for Ecology and Hydrology, Benson Lane, Wallingford, Oxfordshire, OX10 8BB, UK. - S. J. Galswothy, Centre for Mathematical Biology, Mathematical Inst., Univ. of Oxford, 24-29 St Giles, Oxford, OX1 3LB, UK. Present address for SJG: Theoretical Epidemiology, Univ. of Utrecht, Yalelaan 7-9, NL-3584 CL, Utrecht, the Netherlands - P. Manzano, TEG, Depto de Ecología, Univ. Autonóma de Madrid, ES-28049 Madrid, Spain. - P. Poschlod, Inst. of Botany, Univ. Regensburg, DE-93040 Regensburg, Germany. - C. Eichberg, Vegetation Ecology, Inst. of Botany, Darmstadt Univ. of Technology, DE-64287 Darmstadt, Germany. Present address for CE: Faculty of Geography and Geosciences, Geobotany, Univ. of Trier, DE-54296 Trier, Germany.

\begin{abstract}
Studies of external seed transport on animals usually assume that the probability of detachment is constant, so that seed retention should show a simple exponential relationship with time. This assumption has not been tested explicitly, and may lead to inaccurate representation of long distance seed dispersal by animals. We test the assumption by comparing the fit to empirical data of simple, two-parameter functions. Fifty-two data sets were obtained from five published studies, describing seed retention of 32 plant species on sheep, cattle, deer, goats and mice. Model selection suggested a simple exponential function was adequate for data sets in which seed retention was followed for short periods $(<48 \mathrm{~h})$. The data gathered over longer periods (49-219 days) were best described by the power exponential function, a form of the stretched exponential which allows a changing dropping rate. In these cases the power exponential showed that seed dropping rate decreased with time, suggesting that seeds vary in attachment, with some seeds becoming deeply buried or wound up in the animal's coat. Comparison of fitted parameters across all the data sets also confirmed that seeds with adhesive structures have lower dropping rates than those without. We conclude that the seed dropping rate often changes with time during external transport on animals and that the power exponential is an effective function to describe this change. We advise that, to analyse seed dropping rates adequately, retention should be measured over reasonable time periods - until most seeds are dropped - and both the simple and power exponential functions should be fitted to the resulting data. To increase its utility, we provide functions describing the seed dropping rate and the dispersal kernel resulting from the power exponential relationship.
\end{abstract}

The fitting of mathematical functions to dispersal data is a key step towards the understanding and prediction of dispersal, and in using such movement information in models of spatial dynamics (Bullock et al. 2006). Many functions have been used to describe dispersal kernels in general, or with more specialised applications to particular dispersal processes (Bullock et al. 2006, Jongejans et al. 2008). Almost all functions used are phenomenological rather than mechanistic, in the sense that they are not representing the dispersal mechanism, but are simple, flexible formulations which show good statistical fit to some data sets (Jongejans et al. 2008). For this reason, care should always be taken when using such fitted functions to make statements about long-distance dispersal or in spatial models. Truly mechanistic functions are not available for most seed dispersal types, but a step towards improving phenomenological functions would be to base their formulation more explicitly on the specific dispersal process (Bullock et al. 2006). In this paper, we consider such an improvement for the description of seed retention on animals.

A number of studies of the external or internal transport of seeds by animals have used simulation approaches to derive dispersal distances (Pakeman 2001, Mouissie et al. 2005, Westcott et al. 2005, Couvreur et al. 2008, Will and Tackenberg 2008). These combine measured data describing: 1) the proportions of seeds attaching to or ingested by animals; 2) the movement path of the animals; and 3) the dropping rate of seeds from the animals. The animal movement in such simulations is often well characterised, for example using directly observed data (Will and Tackenberg 2008) or well-parameterised correlated random walk models (Mouissie et al. 2005). Such characterisation is critical, as it is generally accepted that the large distances moved by some animals during this process may account for long-distance dispersal events (Nathan et al. 2008). The seed retention process has received less attention, and here we focus on seed 
detachment during external transport on animals (epizoochory). Where dropping rates are considered explicitly, for example in some of the simulation models of epizoochory, most studies assume that the probability of seed detachment from the dispersal vector is constant over time and space (Bullock and Primack 1977, Mouissie et al. 2005, Couvreur et al. 2008). This is a sensible first assumption and would mean that the proportion of seeds remaining attached to the vector follows an exponential function over time (Wichmann et al. 2009). However, the truth of this assumption has not been tested generally. In particular, it may be that the seed dropping rate is not constant, but is a function of time since attachment. Will and Tackenberg (2008) implied this possibility by using a gamma distribution, and Wichmann et al. (2009) explored it more explicitly by considering modifications to the exponential function. Fitting a variety of exponential functions to data for seed dispersal by humans, Wichmann et al. (2009) showed that seed retention probability increased with time since attachment. Because a greater proportion of seeds are retained for longer by the animal than would be allowed by a constant dropping rate, this finding suggests a mechanism which would increase the potential for long-distance seed dispersal.

In this paper, we explore these exponential functions further and determine whether seed dropping rates from animal vectors are constant or change with time since attachment. We do so by analysing a range of published data which describe dispersal of a variety of plant species by external transport on sheep, cattle, goats, deer and mice. In doing so, we present a generic approach to analysing seed dropping rates and suggest that modelling and analysis of epizoochory should allow for more complex seed retention patterns than are generally considered.

\section{Methods}

\section{Exponential functions for seed retention}

A constant seed dropping rate means that the proportion of seeds, of those attached at time 0 , left on the animal vector (lov) at time $t$ follows a simple exponential function (Wichmann et al. 2009),

$\operatorname{lov}(\mathrm{t})=\operatorname{aexp}(-\mathrm{bt})$,

where $\mathrm{a}$ is the proportion of seeds attached at $\mathrm{t}=0$, i.e. 1 , and $\mathrm{b}$ is the (constant) dropping rate of seeds. In developing modifications of the simple exponential to allow for a changing dropping rate with time, both Wichmann et al. (2009) and we aim to develop functions with only two parameters and relatively simple formulations; to allow straightforward model selection and to avoid over-fitting, especially as data sets can be small.

Modifications to the simple exponential involve making the dropping rate a function of time. Wichmann et al. (2009) had most success with, what they called, the power exponential, which is a form of the stretched exponential function (also known as the Kohlrausch-Williams-Watts function), as has been used in many other scientific disciplines (Cardona et al. 2007). $\operatorname{lov}(\mathrm{t})=\operatorname{aexp}\left(-\mathrm{t}^{\mathrm{b}}\right)$

where, again, $\mathrm{a}$ and $\mathrm{b}$ are fitted parameters (for simplicity, we use $a$ and $b$ in all equations, but note that they signify different values for each equation). The dropping rate in general is

$r(t)=\frac{-\frac{d^{2}}{d t^{2}}(1-\operatorname{lov}(t))}{\frac{d}{d t}(1-\operatorname{lov}(t))}$

which becomes for the power exponential

$\mathrm{r}(\mathrm{t})=\mathrm{bt} \mathrm{t}^{\mathrm{b}-1}-\frac{\mathrm{b}-1}{\mathrm{t}}$

and $\mathrm{b}>1$ indicates an increasing dropping rate with time, while $\mathrm{b}<1$ corresponds to a decreasing dropping rate.

Wichmann et al. (2009) also suggested two forms of double exponential function:

$\operatorname{lov}(\mathrm{t})=\operatorname{aexp}(\exp (-\mathrm{bd}))$

which allows a decrease in dropping rate with time, and

$\operatorname{lov}(t)=\operatorname{aexp}(-\exp (b d))$

in which dropping rate increases with time.

In the analyses carried out in this paper, we also use an inverse power function as a comparator for the exponential functions,

$\operatorname{lov}(\mathrm{t})=\mathrm{at}^{-\mathrm{b}}$

This function does not mirror the process of seed dropping over time as do the exponential functions. It is also not a true probability distribution, which limits its utility (Bullock et al. 2006). However the inverse power is a simple yet flexible two-parameter function allowing a long tail, which is being used widely in dispersal studies (Sundberg 2005, Mundt et al. 2009, Stevens et al. 2010), and has shown good fit to data for seed retention during both external (Manzano and Malo 2006) and internal (Clark et al. 2005) transport on animals.

\section{Data sets for seed retention on animals}

There are relatively few studies of seed retention on animals which cover adequate time periods and provide measures of seed numbers at sufficient time points to allow alternative functions to be compared with confidence. We used a number of studies for which we could obtain the data and which, to a varying degree, fulfilled these quality criteria. In the descriptions below we use the time units reported for each study. We subsequently refer to these datasets by the name of the first author. Each author indicated the degree to which the seeds of each species had structures which might promote adhesion to animal vectors. We used this information, explained in more detail below for each paper, to classify species into two classes: with or without adhesive structures (Table 1). 
Table 1. AIC values for functions fitted to data sets describing seed retention on a range of animal vectors. Where the AIC indicates a single best fitting function (Manzano, Fischer, Eichberg), its AIC is in bold. In other cases (Kiviniemi, Shmida) the AIC values do not indicate a single best fit among the exponential functions, so the simple exponential is highlighted as the simplest model among those fitting best. Superscripts indicate seeds A with or N without adhesive structures (see text).

\begin{tabular}{|c|c|c|c|c|c|c|c|}
\hline \multirow[b]{2}{*}{ Species } & \multicolumn{5}{|c|}{$\mathrm{AIC}$} & \multicolumn{2}{|c|}{$\begin{array}{l}\text { Parameters of selected } \\
\text { function (in bold) }\end{array}$} \\
\hline & $\begin{array}{l}\text { Simple } \\
\exp \end{array}$ & $\begin{array}{l}\text { Power } \\
\text { exp }\end{array}$ & $\begin{array}{l}\text { Double } \\
\exp (1)\end{array}$ & $\begin{array}{l}\text { Double } \\
\exp (2)\end{array}$ & $\begin{array}{l}\text { Inv. } \\
\text { power }\end{array}$ & a & $\mathrm{b}$ \\
\hline \multicolumn{8}{|c|}{ Manzano and Malo (2006). Sheep. Maximum time: 3548 h (148 days) } \\
\hline Daucus carotaA & 49.9 & 36.0 & 48.0 & 52.3 & 54.1 & 1.152 & 0.164 \\
\hline Hordeum murinum ${ }^{\mathrm{A}}$ & 47.5 & 30.5 & 43.5 & 49.6 & 45.3 & 1.111 & 0.180 \\
\hline Plantago lagopus ${ }^{\mathrm{N}}$ & 44.5 & 26.5 & 41.6 & 47.6 & 40.7 & 1.083 & 0.208 \\
\hline Trifolium angustifolium ${ }^{\mathrm{A}}$ & 66.6 & 57.7 & 64.5 & 67.0 & 86.4 & 1.435 & 0.086 \\
\hline \multicolumn{8}{|c|}{ Fischer et al. (1996). Sheep. (fleece thickness). Maximum time: 49 days (1176 h) } \\
\hline Bromus erectus ${ }^{\mathrm{A}}(3 \mathrm{~cm})$ & 20.7 & 14.6 & 20.3 & 21.0 & 23.8 & 1.039 & 0.283 \\
\hline B. $\operatorname{erectus}^{\mathrm{A}}(5 \mathrm{~cm})$ & 22.9 & 16.7 & 22.7 & 23.4 & 28.3 & 1.071 & 0.233 \\
\hline Helianthemum nummularium $\mathrm{N}(3 \mathrm{~cm})$ & 15.2 & 9.7 & 14.4 & 15.4 & 18.8 & 1.080 & 0.223 \\
\hline H. nummularium ${ }^{\mathrm{N}}(5 \mathrm{~cm})$ & 16.7 & 10.9 & 15.5 & 16.9 & 20.5 & 1.123 & 0.187 \\
\hline \multicolumn{8}{|c|}{ Kiviniemi and Telenius (1998). Yellow-necked mice Apodemus flavicollis. Maximum time: 9 min $40 \mathrm{~s}$} \\
\hline Filipendula vulgaris ${ }^{\mathrm{A}}$ & 21.3 & 25.9 & 28.2 & 24.3 & 34.1 & 0.982 & 0.167 \\
\hline Seseli libanotis ${ }^{\mathrm{A}}$ & 7.5 & 9.0 & 10.5 & 7.7 & 12.6 & 1.000 & 0.056 \\
\hline Daucus carotaA & 28.0 & 37.3 & 35.1 & 28.1 & 50.1 & 0.999 & 0.008 \\
\hline Geum rivale ${ }^{\mathrm{A}}$ & 28.9 & 37.3 & 35.6 & 32.1 & 42.3 & 0.769 & 0.009 \\
\hline Torilis japonica ${ }^{\mathrm{A}}$ & 21.7 & 28.0 & 29.6 & 24.3 & 37.1 & 1.000 & 0.135 \\
\hline Sanicula europaA & 22.1 & 28.0 & 28.5 & 23.9 & 37.1 & 1.000 & 0.013 \\
\hline Agrimonia eupatoria ${ }^{\mathrm{A}}$ & 15.1 & 16.1 & 19.0 & 16.7 & 21.9 & 0.888 & 0.023 \\
\hline Ranunculus acris ${ }^{\mathrm{N}}$ & 9.6 & 9.2 & 12.3 & 11.3 & 12.8 & 1.000 & 0.051 \\
\hline Anthriscus sylvestris ${ }^{\mathrm{N}}$ & 8.0 & 7.2 & 10.4 & 9.7 & 10.1 & 1.000 & 0.072 \\
\hline Potentilla reptans ${ }^{\mathrm{N}}$ & 13.0 & 16.3 & 16.7 & 13.7 & 22.0 & 1.000 & 0.023 \\
\hline Polygala vulgaris ${ }^{\mathrm{N}}$ & 14.1 & 17.8 & 16.8 & 14.1 & 24.7 & 1.000 & 0.017 \\
\hline Triglochin palustre ${ }^{N}$ & 7.6 & 9.2 & 10.0 & 7.6 & 12.7 & 1.000 & 0.053 \\
\hline Carum carvi $\mathrm{N}$ & 7.3 & 8.7 & 9.9 & 7.4 & 12.1 & 1.000 & 0.058 \\
\hline \multicolumn{8}{|c|}{ Kiviniemi and Telenius (1998). Fallow deer Dama dama. Maximum time: 2 h 45 min } \\
\hline Geum rivale $\mathrm{A}^{\mathrm{A}}$ & 11.2 & 13.3 & 13.2 & 11.5 & 18.2 & 1.000 & 0.0003 \\
\hline Agrimonia eupatoria ${ }^{\mathrm{A}}$ & 8.4 & 9.4 & 10.8 & 8.9 & 12.7 & 1.000 & 0.0006 \\
\hline Triglochin palustre ${ }^{\mathrm{N}}$ & 11.7 & 13.3 & 13.2 & 12.1 & 18.3 & 1.000 & 0.0003 \\
\hline \multicolumn{8}{|c|}{ Kiviniemi and Telenius (1998). Cattle. Maximum time: 3 h 15 min } \\
\hline Filipendula vulgaris ${ }^{\mathrm{A}}$ & 7.3 & 8.6 & 9.6 & 8.1 & 12.1 & 1.000 & 0.002 \\
\hline Geum rivale ${ }^{\mathrm{A}}$ & 8.7 & 12.7 & 13.8 & 10.2 & 17.3 & 1.000 & 0.0008 \\
\hline Agrimonia eupatoriaA & 10.6 & 16.5 & 15.5 & 11.4 & 22.1 & 1.000 & 0.0004 \\
\hline Ranunculus acris $\mathrm{N}$ & 7.1 & 7.9 & 9.9 & 8.3 & 11.3 & 1.000 & 0.003 \\
\hline Anthriscus sylvestris ${ }^{\mathrm{N}}$ & 5.8 & 6.2 & 8.1 & 8.8 & 9.0 & 1.000 & 0.007 \\
\hline Polygala vulgaris $\mathrm{N}$ & 14.1 & 17.8 & 16.8 & 14.1 & 24.7 & 1.000 & 0.003 \\
\hline Triglochin palustre ${ }^{\mathrm{N}}$ & 7.6 & 9.2 & 10.0 & 7.6 & 12.7 & 1.000 & 0.004 \\
\hline Carum carviN & 7.3 & 8.7 & 9.9 & 7.4 & 12.1 & 1.000 & 0.005 \\
\hline \multicolumn{8}{|c|}{ Eichberg et al. (2005). Sheep. Maximum time: 5256 h (219 days) } \\
\hline Jurinea cyanoides ${ }^{\mathrm{A}}$ & 39.9 & 25.8 & 34.3 & 40.9 & 36.1 & 1.036 & 0.251 \\
\hline \multicolumn{8}{|c|}{ Shmida and Ellner (1983). Sheep. Maximum time: $48 \mathrm{~h}$} \\
\hline Cynoglossum creticum ${ }^{\mathrm{A}}$ & 7.9 & 8.3 & 8.0 & 7.9 & 16.9 & 0.958 & 0.010 \\
\hline Medicago minimaA & 10.5 & 9.2 & 9.5 & 9.7 & 15.6 & 1.000 & 0.028 \\
\hline Picris galilaea ${ }^{\mathrm{A}}$ & 9.5 & 9.7 & 10.2 & 9.4 & 13.7 & 0.773 & 0.052 \\
\hline Scorpiurus muricatus ${ }^{\mathrm{A}}$ & 9.6 & 9.8 & 10.1 & 9.5 & 15.2 & 0.833 & 0.026 \\
\hline Torilis leptophylla & 9.8 & 9.2 & 9.4 & 9.8 & 13.6 & 0.725 & 0.036 \\
\hline Onobrychis squarrosa ${ }^{N}$ & 9.5 & 8.1 & 9.9 & 8.5 & 11.5 & 1.000 & 0.226 \\
\hline Biscutella didymaN & 9.8 & 7.9 & 9.9 & 8.5 & 10.8 & 1.000 & 0.226 \\
\hline Scorzonera papposa ${ }^{\mathrm{N}}$ & 8.9 & 8.3 & 10.0 & 8.8 & 11.5 & 0.658 & 0.093 \\
\hline Trifolium stellatum ${ }^{\mathrm{N}}$ & 9.2 & 8.9 & 9.1 & 9.4 & 13.2 & 0.787 & 0.054 \\
\hline Aegilops peregrina ${ }^{\mathrm{A}}$ & 9.1 & 9.5 & 8.9 & 9.2 & 14.9 & 0.892 & 0.035 \\
\hline Avena sterilis ${ }^{\mathrm{A}}$ & 9.2 & 9.4 & 9.0 & 9.3 & 14.9 & 0.874 & 0.031 \\
\hline Crithopsis delileana ${ }^{\mathrm{A}}$ & 8.5 & 9.2 & 8.3 & 8.5 & 16.1 & 0.969 & 0.022 \\
\hline Bromus fasciculatus ${ }^{N}$ & 8.4 & 8.7 & 9.0 & 8.9 & 13.0 & 0.866 & 0.079 \\
\hline Trachynia distachya ${ }^{\mathrm{N}}$ & 7.8 & 6.8 & 8.9 & 8.5 & 10.5 & 1.000 & 0.029 \\
\hline \multicolumn{8}{|c|}{ Shmida and Ellner (1983). Goats. Maximum time: $48 \mathrm{~h}$} \\
\hline Cynoglossum creticum ${ }^{\mathrm{A}}$ & 8.0 & 9.6 & 8.6 & 8.4 & 14.4 & 0.988 & 0.062 \\
\hline Medicago minima ${ }^{\mathrm{A}}$ & 8.6 & 9.6 & 8.6 & 8.9 & 15.0 & 0.951 & 0.040 \\
\hline Scorpiurus muricatus ${ }^{\mathrm{A}}$ & 7.8 & 7.8 & 9.1 & 8.2 & 12.0 & 0.871 & 0.131 \\
\hline Torilis leptophylla ${ }^{\mathrm{A}}$ & 7.1 & 6.8 & 9.0 & 8.7 & 10.8 & 1.000 & 0.321 \\
\hline Trifolium stellatum ${ }^{\mathrm{N}}$ & 7.7 & 6.5 & 8.8 & 8.5 & 10.0 & 1.000 & 0.349 \\
\hline
\end{tabular}




\section{Manzano and Malo (2006)}

Between 36-150 marked seeds of three forbs and one grass species (Table 1) were pressed into the fleeces of each of five merino sheep during the annual transhumance in central Spain. Frequent counts were made of seeds remaining up to a maximum of $3548 \mathrm{~h}$ (148 days) later. We used the individual data for each of the five wethers in our analyses. The authors did not classify the species formally, but noted that all except Plantago lagopus had seeds with adhesive structures.

\section{Fischer et al. (1996)}

270 marked seeds of a grass and a forb species (Table 1) were placed on each of two ewes - one with a $3 \mathrm{~cm}$ fleece length and the other with a $5 \mathrm{~cm}$ fleece - grazing on calcareous grasslands in southwestern Germany. Frequent counts of seeds remaining in the fleece were made up to a maximum of 49 days. We used the original data for our analyses, which were done separately for each sheep. The two species were chosen by the authors to represent seeds with and without adhesive structures.

\section{Kiviniemi and Telenius (1998)}

This paper provides three data sets which we extracted from the published graphs. Seeds of 13 forb species (Table 1) were placed on the backs of three yellow-necked mice Apodemus flavicollis put into indoor enclosures. Seeds were placed individually and the retention time of each was measured in seconds - the maximum retention time being $580 \mathrm{~s}$ (9 min $40 \mathrm{~s}$ ). We used the data as reported - the mean proportion of seeds remaining attached at $10 \mathrm{~s}$ time steps (Fig. 1 in Kiviniemi and Telenius 1998).

The same paper gives graphs from studies in Swedish pastures (Kiviniemi 1996) of mean proportions of seeds of three forb species (Table 1) retained on fallow deer Dama dama at $900 \mathrm{~s}$ intervals up to $9900 \mathrm{~s}(165 \mathrm{~min})$ and of eight forbs (Table 1) retained on domestic cattle at 100-900 s intervals up to $11700 \mathrm{~s}$ (195 min) (Fig. $2 \mathrm{~b}$ and $2 \mathrm{c}$ respectively in Kiviniemi and Telenius 1998). The authors investigated the seeds of all species used and classified those with hairs, hooks or spikes as having adhesive structures compared to those with no obvious structures.

\section{Eichberg et al. (2005)}

Achenes of the forb Jurinea cyanoides were placed into the fleeces of four Rhoen sheep (180 achenes per sheep) and counted at frequent intervals up to $311 \mathrm{~h}$ later, and again after 7 months $(5256 \mathrm{~h})$. The study took place in calcareous sand pioneer grasslands of southcentral Germany, and we used the individual data for each of the four wethers in our analyses. Jurinea cyanoides seeds have bristles and teeth which probably aid adhesion.

\section{Shmida and Ellner (1983)}

Marked seeds of 13 forbs and six grasses were placed on pastoral livestock grazing on Israeli Mediterranean chaparral. These comprised three sheep (40 seeds per sheep of each species) and three goats (20 seeds per goat of each species) and seed numbers remaining were counted at six intervals up to $48 \mathrm{~h}$ later. We analysed data given in the paper (Table III in Shmida and Ellner 1983) of summed numbers across the three replicate animals. To allow us to compare function fits effectively, we only analysed those species for which there were non-zero data at more than three time points (14 of the sheep data sets, and five of the goat data sets; Table 1). The authors classified their species into four types based on the development of characters which might promote adhesion, from type 0 with no appendages to type 3 with dense hooked hairs or bristles. We classified their types 0 and 1 as being without adhesive structures and type 3 and having such structures (none of the species we analysed were in type 2).

\section{Statistical fitting of functions}

All five functions - simple exponential, power exponential, double exponential 1 (decreasing dropping rate), double exponential 2 (increasing dropping rate), and the inverse power - were fitted to each data set by generalized non-linear modelling through Proc NLMIXED in SAS using a binomial distribution.

The lov-values were calculated as proportions of the number of seeds originally attached to the vector. The time units (days, h or s) used in the original papers were retained as a reminder of differences among the studies, and any zero values were changed to $10^{-5}$ to avoid fitting problems. Akaike's information criterion (AIC) was used to compare the fits of the different functions to each data set and plots of the fitted function were compared to the retention data.

The classification of species as being with or without seed structures which might promote adhesion to the animal allowed us to determine whether retention was enhanced by adhesive structures. Excluding the Eichberg et al. (2005) study which used only one species, we divided data into seven 'groups' classified by the study and, where more than one vector was included into a study, further by the vector: Manzano, Fischer, Kiviniemi-mice, Kiviniemi-deer, Kiviniemi-cattle, Shmida-sheep, Shmida-goats. The data sets in each group all had a single selected function, either the simple exponential or the power exponential (Table 1). Thus, retention differences among species within a group could be analysed using the fitted b parameter, for which a lower value indicates longer retention times in both functions. Including 'group' as a random factor to account for differences among groups because of the study design, the vector and the selected exponential function, we tested for differences in the value of $b$ between species with and without adhesive structures using ANOVA. The b values were $\log _{\mathrm{e}}$ transformed, which achieved normality of residuals.

\section{Results}

The Manzano, Fischer and Eichberg studies, which measured seed retention on sheep over many tens of days, produced data sets that were all best fitted by the power exponential function (Table 1), whose fit was generally much better than that of the simple exponential. This pattern was clear for the Manzano and Eichberg data sets, where the difference in AIC $(\triangle \mathrm{AIC})$ between the power and simple exponentials was at least 9 and the fitted power exponential function described the data well (Fig. 1a, e). Burnham and Anderson (2002) recommend that $\triangle \mathrm{AIC}<2$ would suggest little difference in fit 

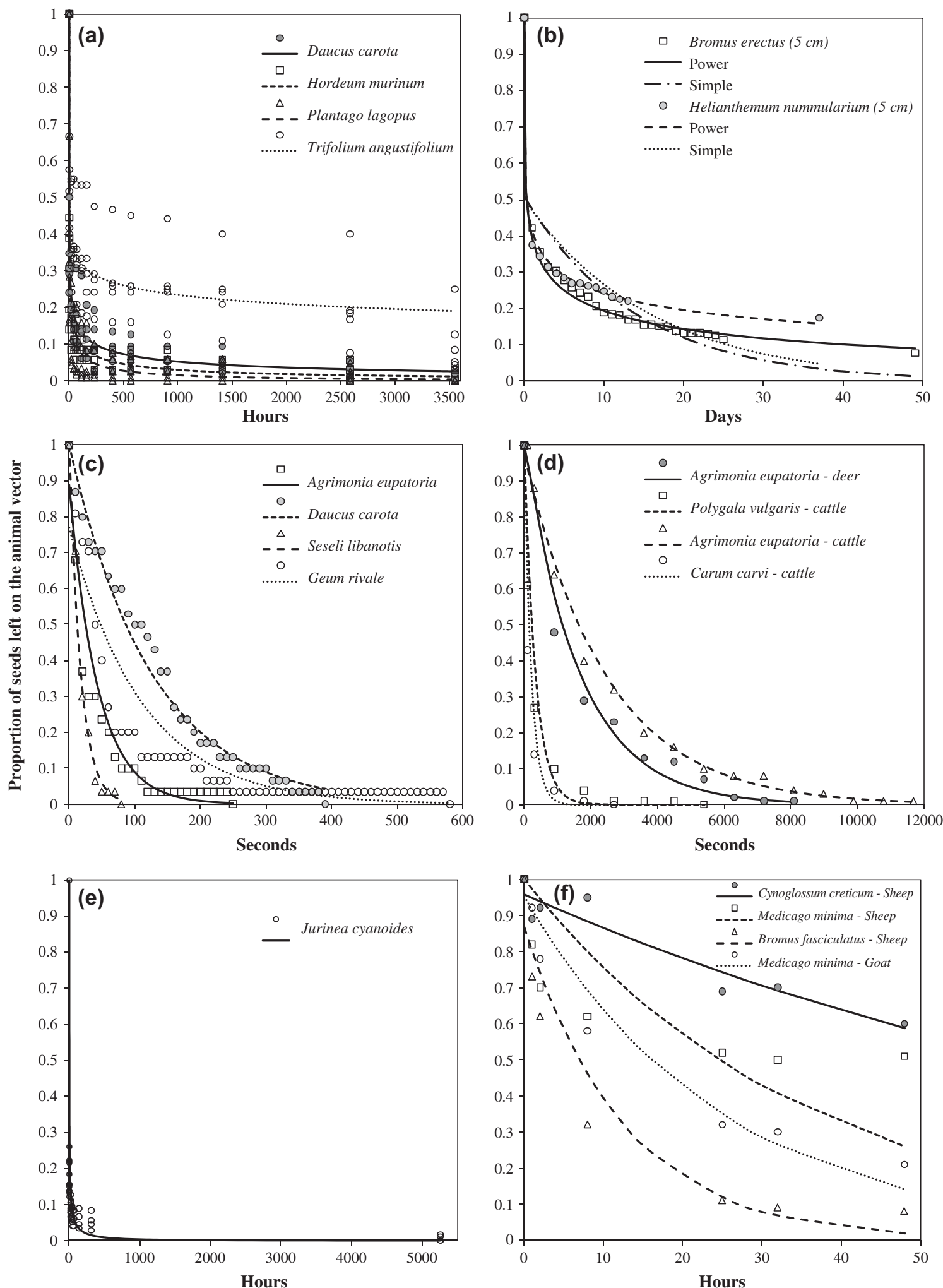

Figure. 1. Example plots of data and the functions selected following model comparison for species from each of the data sets analysed (Table 1). (a) All data sets from Manzano and Malo (2006), for which the power exponential was the best fit for all species. (b) Two data sets from Fischer et al. (1996), contrasting the power exponential, which was the best fit, and the simple exponential. (c) Kiviniemi and Telenius (1998) data for attachment to wood mice, illustrating the selected simple exponential. (d) Kiviniemi and Telenius (1998) data for attachment to cattle and deer, illustrating fits of the simple exponential. (e) Eichberg et al. (2005) data for Jurinea cyanoides, which reflected the power exponential. (f) Data from Shmida and Ellner (1983), described by the simple exponential. The differences in time units among the plots reflect the units used in the original studies. 

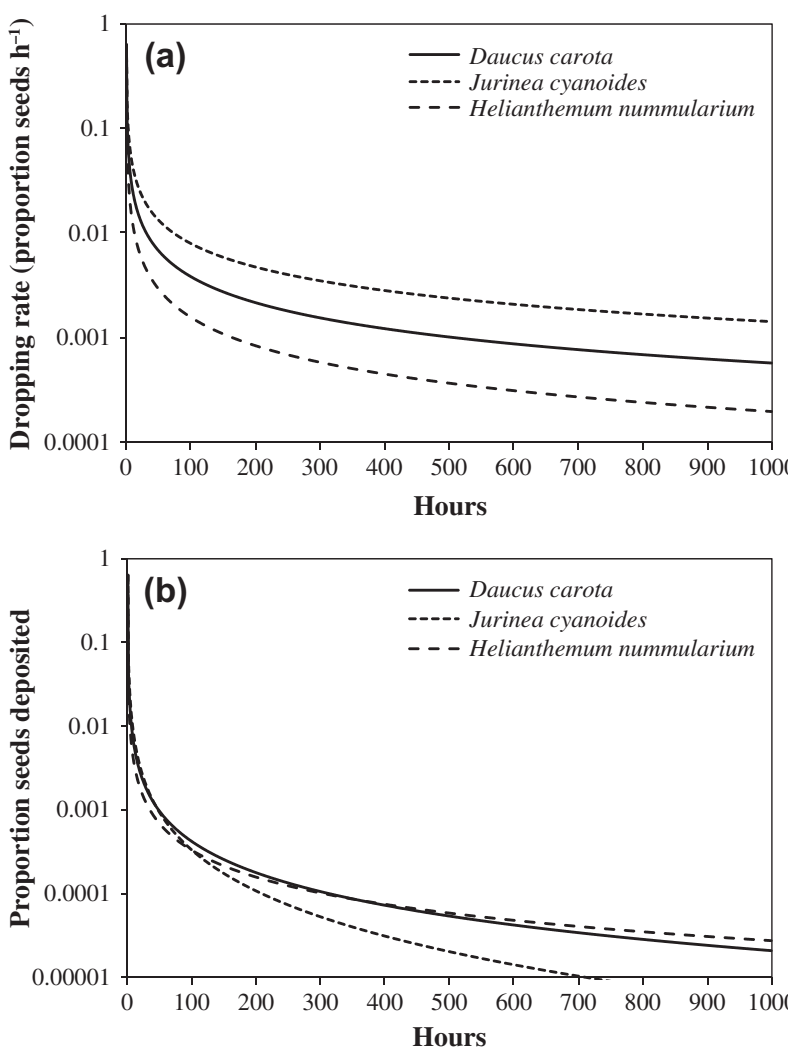

Figure 2. Plots illustrating the consequences of the power exponential function, using a single species from each of the three data sets for which seed retention was followed for long time periods: Daucus carota (Manzano and Malo 2006); Helianthemum nummularium (on $5 \mathrm{~cm}$ fleece, Fischer et al. 1996 - the power exponential was re-calculated for data in hour units); and Jurinea cyanoides (Eichberg et al. 2005). (a) The proportion of remaining seeds dropping from the vector over each hour, illustrating the change in dropping rate with time. (b) The dispersal kernels resulting from the fitted power exponential, showing the distribution of deposited seeds with time since attachment.

between functions. Larger $\triangle \mathrm{AIC}$ values indicate better support for the function with the lower AIC, although values of $\triangle \mathrm{AIC}=4-7$ are interpreted as giving only moderate support. For the Fischer data sets the power exponential always had the lowest AIC although $\triangle \mathrm{AIC}=5-7$ compared to the simple exponential. However, comparison of the curves described by the fitted simple and power exponential functions showed the latter matched the Fischer data more accurately than the former (Fig. 1b). The AIC for the power exponential was also always lower than those for the two double exponentials for the Manzano, Fischer and Eichberg data sets with $\triangle \mathrm{AIC}$ $=5-21$, and the relative fit of the inverse power was usually even worse ( $\triangle \mathrm{AIC}=9-28)$. Furthermore, one would expect a $\sim 1$ for the simple and power exponentials, as this is the value of lov at $t=0$ (this is not the case for the double exponentials or the inverse power). Indeed, a $\sim 1$ for the fitted power exponential functions in the Manzano, Fischer and Eichberg datasets (Table 1).

A quite different story emerges from the Kiviniemi and Shmida data sets, which represent retention over short time periods from a few minutes to $48 \mathrm{~h}$. Here, the simple exponential was always among the best fitting models. For each of these data sets the AIC of simple exponential was either the lowest or its $\triangle \mathrm{AIC}$ compared to the function with the lowest AIC was $<2$. However, in every case at least one of the other exponential functions had a $\triangle$ AIC $<$ 3 compared to the simple exponential. In addition, plots showed that the fitted simple exponential functions in general described the data well (Fig. 1b-d), although the lack of flexibility was apparent in the occasional rather poor descriptions of certain data sets, such as Geum rivale in Fig. 1c and Medicago minina by sheep in Fig. 1f. Therefore, we selected the simple exponential as the simplest of the most likely functions describing the Kiviniemi and Shmida data sets. The fitted simple exponential for these data sets also had the expected a $\sim$ (Table 1 ). The inverse power showed the worst fit, having the highest AIC for all but one of 43 Kiviniemi and Shmida data sets and the $\triangle \mathrm{AIC}$ compared to the simple exponential ranged between 1 and 22 (median $=5)$.

As indicated by Eq. 4, the dropping rate (i.e. the proportion of remaining seeds which detach from the animal per unit time) is a function of time since attachment, and example plots of fitted dropping rates for a species from each of the Manzano, Fischer and Eichberg data sets are given in Fig. 2a. This illustrates the critical difference to the constant dropping rate which is the basis of the simple exponential; the power exponential has a dropping rate which decreases rapidly over the early time periods, but becomes small and changes more slowly as time progresses. The function can be used to derive a dispersal kernel, as in Fig. 2b, which shows the proportion of seeds deposited over each hour time step for the example species.

ANOVA indicated strong differences among the study/ vector groups in the value of $b$ ('group' factor $F_{6,43}=43$, $\mathrm{p}<0.001$ ), but also that after accounting for these differences, $\mathrm{b}$ was lower for species having adhesive structures (least squares mean $=0.019$ ) than those without (least squares mean $\left.=0.039 ; \mathrm{F}_{1,43}=11, \mathrm{p}<0.01\right)$. This indicates that adhesive structures increased retention times of seed across the studies analysed.

\section{Discussion}

Our analysis gave clear results that the power exponential was the better function than the simple exponential for describing seed retention on animal vectors where retention was measured over long time periods. This suggests that dropping probability often decreases with time since attachment (i.e. $\mathrm{b}<<1$ ), to quite a marked degree. This finding mirrors our earlier analysis of dispersal of two Brassica species on human shoes, for which the power exponential also described retention better than alternative exponential formulations (Wichmann et al. 2009).

It is informative that the power exponential was the best fit in the three data sets in which seed retention was measured over substantial time periods: Manzano and Malo (2006) for 3548 h; Fischer et al. (1996) for 1176 h; and Eichberg et al. (2005) for $5256 \mathrm{~h}$. We also note that these studies were all carried out with sheep. The Kiviniemi and Telenius (1998) study, for which the simple exponential function was the best description, were carried out on short-haired animals - 
mice, cattle and deer. It maybe, therefore, that a decreasing dropping rate with time is more likely on animals with thick or woolly coats. Such a conclusion can only be tentative as none of the individual studies contrasted smooth-coated and woolly animals over adequate time periods. Furthermore, Wichmann et al. (2009) found the same dropping rates pattern for seeds on the shoes of humans. However, the power exponential indicates that some seeds are more strongly attached to the vector than others, for example by becoming deeply buried or wound up in the animal's coat, and this is more likely in thick-coated animals. If some of these seeds are so well attached that they are effectively stuck in the coat and will never drop out, then the formulation of the seed retention function would be more accurate if it included a parameter describing this stuck proportion (Will and Tackenberg 2008), especially as these seeds cannot contribute to effective dispersal. Among the 52 data sets used here there is little evidence that seeds do become permanently stuck. In all data sets except four either no seeds remained attached by the final time point or seed dropping continued between the penultimate and final time points.

The Shmida and Ellner (1983) study followed seed retention on sheep and goats for $48 \mathrm{~h}$ and we found that no functions fitted the data better than the simple exponential. In this case, especially for sheep, substantial proportions of seeds often remained on the animals after 48 h (Fig. 1f). Thus, while this study was pioneering and important, it also illustrates that an adequate time period is required to characterise the pattern of long-term seed retention and thus determine whether the dropping rate is constant or otherwise.

We tested the two forms of double exponential as they seemed sensible alternatives to the power exponential (Wichmann et al. 2009). The increasing double exponential function (Eq. 6) never showed a better fit than the simple exponential, confirming the conclusion that seed dropping rate is either constant or decreases with distance. The decreasing double exponential (Eq. 5) seems not to describe well the retention process; even in the Manzano, Fischer and Eichberg data sets, which were described better by the power than the simple exponential, this double exponential had a $\triangle$ AIC $>4$ compared to the simple exponential in only two of the nine data sets. The formulation of the inverse power is not linked simply to the retention process, but it is extremely popular in dispersal studies, both of seed retention (Clark et al. 2005, Manzano and Malo 2006), and more generally because it can describe well dispersal data with long tails (Bullock et al. 2006). However, the inverse power was much worse than the power exponential at describing the Manzano, Fischer and Kiviniemi retention data. If we add to this finding the mathematical problems of the inverse power, it is a fair conclusion that the inverse power is not useful in describing seed retention.

This paper illustrates why it is important to fit multiple functions to dispersal data (Bullock et al. 2006). Our analyses suggest that it will always be informative to fit at least the simple and power exponential functions to seed retention data. Indeed, if one wanted to compare only these two alternative functions, a single three parameter function could be used, which is the full version of the stretched exponential: $\operatorname{lov}(t)=\operatorname{aexp}\left(-b t^{c}\right)$

where $\mathrm{c}$ is the stretching exponent and will reduce to 1 if the simple exponential is the better fit. Indeed, to reduce the risk of over-fitting, the parameter a could also be removed as in the simple and power exponential $\mathrm{a}$ is the proportion of seeds attached at $\mathrm{t}=0$, which will always be 1 . We retained $\mathrm{a}$ in this paper because this interpretation of a does not hold for the other functions tested and because we were more interested in fitting the tail of the curve, which is governed by $b$.

The simple exponential is easy to use in models of seed dispersal by external transport on animals, whereby the constant dropping rate can be combined with information on animal movement (Mouissie et al. 2005, Couvreur et al. 2008). The power exponential introduces minor complications. If animals move such that they show a constant rate of displacement then the dispersal kernel is simply this displacement rate multiplied into the probability density function of the power exponential with respect to time,

$$
\begin{aligned}
f(t) & =\frac{d}{d t}(1-\operatorname{lov}(t)) \\
& =a b t^{b-1} \exp \left(-t^{b}\right)
\end{aligned}
$$

which is a Weibull distribution with shape parameter $b$ and scale parameter 1. Again, this simplifies is a is taken as 1 . If movement is more complex then the measured (Will and Tackenberg 2008) or modelled (e.g. using correlated random walks; Mouissie et al. 2005) movement path can be used with the time-dependent dropping rate (Eq. 4). Dispersal kernels generated using the power exponential will be more leptokurtic than those using a simple exponential retention function, as a greater proportion of seeds will be dropped at early times, but a small proportion of seeds will remain attached for relatively long time periods. This small proportion of seeds could be important in increasing the potential for long-distance dispersal.

A smaller value for the $b$ parameter in both the simple and power exponential functions indicates a lower dropping rate (Eq. 1, 4). We were fortunate that each paper included an assessment of the presence of structures on the seeds of each species which might enhance adhesion to animal vectors. Thus, we were able to use the fitted $b$ values to ask whether to presence of adhesive structures on seeds increases seed retention. Both Shmida and Ellner (1983) and Kiviniemi and Telenius (1998) found positive answers to this question using less formal analyses and we confirmed a clearly decreased $b$ for models fitted to species with adhesive seeds over all studies and vectors. This suggests an extra utility of the exponential functions in allowing comparison and synthesis across different studies.

In conclusion, we have re-analysed a range of data sets which describe measured seed retention on animals and have shown that where the dropping rate of seeds is measured over adequate periods it is often not constant, but decreases as time passes. The power exponential function describes this decrease well and better than alternative functions. These results indicate that the common assumption of a constant dropping rate could often lead to erroneous predictions in models of seed dispersal by external transport on animals. 
We suggest that the power exponential is used to analyse seed retention data in order to test such assumptions and to increase accuracy of seed dispersal modelling.

Acknowledgements - We thank Steve Freeman and Rosie Hails for advice. This work was funded by NERC grant NE/B503141/1.

\section{References}

Bullock, J. M. et al. 2006. Measuring plant dispersal: an introduction to field methods and experimental design. - Plant Ecol. 186: 217-234.

Bullock, S. H. and Primack, R. B. 1977. Comparative experimental study of seed dispersal on mammals. - Ecology 58: 681-686.

Burnham, K. P. and Anderson, D. R. 2002. Model selection and inference: a practical information-theoretic approach. - Springer.

Cardona, M. et al. 2007. The history of the stretched exponential function. - Ann. Physik 16: 842-845.

Clark, C. J. et al. 2005. Comparative seed shadows of bird-, monkeyand wind-dispersed trees. - Ecology 86: 2684-2694.

Couvreur, M. et al. 2008. Epizoochory by large herbivores: merging data with models. - Basic Appl. Ecol. 9: 204-212.

Eichberg, C. et al. 2005. Epizoochorous and post-dispersal processes in a rare plant species: Jurinea cyanoides (L.) Rchb. (Asteraceae). - Flora 200: 477-489.

Fischer, S. F. et al. 1996. Experimental studies on the dispersal of plants and animals on sheep in calcareous grasslands. - J. Appl. Ecol. 33: 1206-1222.

Jongejans, E. et al. 2008. Dispersal, demography and spatial population models for conservation and control management. - Persp. Plant Ecol. Evol. Syst. 9: 153-170.
Kiviniemi, K. 1996. A study of adhesive seed dispersal of three species under natural conditions. - Acta Bot. Neer. 45: 73-83.

Kiviniemi, K. and Telenius, A. 1998. Experiments on adhesive dispersal by wood mouse: seed shadows and dispersal distances of 13 plant species from cultivated areas in southern Sweden. - Ecography 21: 108-116.

Manzano, P. and Malo, J. E. 2006. Extreme long-distance seed dispersal via sheep. - Front. Ecol. Environ. 4: 244-248.

Mouissie, A. M. et al. 2005. Estimating adhesive seed-dispersal distances: field experiments and correlated random walks. - Funct. Ecol. 19: 478-486.

Mundt, C. C. et al. 2009. Long-distance dispersal and accelerating waves of disease: empirical relationships. - Am. Nat. 173: 456-466.

Nathan, R. et al. 2008. Mechanisms of long-distance seed dispersal. - Trends Ecol. Evol. 23: 638-647.

Pakeman, R. J. 2001. Plant migration rates and seed dispersal mechanisms. - J. Biogeogr. 28: 795-800.

Shmida, A. and Ellner, S. 1983. Seed dispersal on pastoral grazers in open mediterranean chaparral, Israel. - Isr. J. Bot. 32: 147-159.

Stevens, V. M. et al. 2010. A meta-analysis of dispersal in butterflies. - Biol. Rev. 85: 625-642.

Sundberg, S. 2005. Larger capsules enhance short-range spore dispersal in Sphagnum, but what happens further away? - Oikos 108: 115-124.

Westcott, D. A. et al. 2005. Incorporating patterns of disperser behaviour into models of seed dispersal and its effects on estimated dispersal curves. - Oecologia 146: 57-67.

Wichmann, M. C. et al. 2009. Human-mediated dispersal of seeds over long distances. - Proc. R. Soc. B 276: 523-532.

Will, H. and Tackenberg, O. 2008. A mechanistic simulation model of seed dispersal by animals. - J. Ecol. 96: 1011-1022. 